\title{
Strategic Analysis of the Advanced Computing Infrastructure and Future Directions
}

\author{
${ }^{1}$ Aisling Yue Irwing and ${ }^{2}$ Alen Macline \\ ${ }^{1,2}$ Faculty of Engineering and Computing, Dublin City University, Whitehall, Dublin 9, Ireland. \\ 1yueirwingdubline@gmail.com
}

\begin{abstract}
Article Info
Journal of Computing and Natural Science (http://anapub.co.ke/journals/jens/jens.html)

Doi: https://doi.org/10.53759/181X/JCNS202101012

Received 18 December 2020; Revised form 25 January 2021; Accepted 22 March 2021.

Available online 05 July 2021.

(2)21 Published by AnaPub Publications.
\end{abstract}

\begin{abstract}
This paper provides a critical evaluation of the Advanced Computing Infrastructure (ACI), which is a fundamental element of the Cyber Infrastructure (CI) $21^{\text {st }}$ of the Century. Whereas the infrastructure focuses widely on the cyber infrastructure required by education, engineering, and science communities to handle more complex issues and problems, the ACI particularly concentrates on ensuring these societies have ready accessibility to the required advanced computing capacities. The CI of the $21^{\text {st }}$ Century and its framework integrate other key complementariness; however, it integrates overlapping elements: scientific instruments, Computational Data-enhanced Engineering \& Science (CDE\&S), computational science, grand challenge societies, workforce development, Cyber Security (CS), campus bridging, software and data. Various elements were addressed in the CI of the 21st Century in the past decade and processes are in progress to design strategic plans focusing on the different elements.
\end{abstract}

Keywords - Advanced Computing Infrastructure (ACI), Cyber Infrastructure (CI), Cyber Security (CS), Computational Dataenhanced Engineering \& Science $(\mathrm{CDE} \& \mathrm{~S})$

\section{INTRODUCTION}

For nearly four decades, the National Science Foundation (NSF) has been a global leader in high-performance computing, utilization, education and research. NSF calls for new visions, strategies and tactics to accelerate and support an advanced computer infrastructure to make transformative ideas using innovative strategies and approaches that accelerate progress in compute and related technology. In addition, the visions, strategies and tactics lead to exponential growth and complexity of data for science, technology, and education companies. The ACI Strategic Plan outline aims at positioning and supporting the full spectrum of NSF-sponsored societies at the forefront of technology of advanced computer, software and hardware technologies. The project intends to enhance a more supplementary, exhaustive, and structured portfolio of Advanced Computing Infrastructure (ACI) and education and research programs that support the international science, technology, and academic society through the provision of multidisciplinary computer science and technology.

The vision and strategies presented in this section arise from the many discussions held within the NSF and the input of community experts, such as those from the six reporting tasks Force of the Cyber Infrastructure (CI) Consultative Committee and the wide-range Advisory Committees of the Directorate. In order to facilitate state-ofthe-art basic research into Computational Data-enhanced Engineering \& Science (CDE\&S), the NSF will be the leader and will implement an effective portfolio of ACI initiatives and other resources [1]. NSF will also focus on developing its leadership objectives too enhance capital development for humans and CDE\&S training for the benefit of engineering and science fields.

The ACI strategies are based on:

- Firstly, the basic research to completely exploit the concurrency and parallelism through technologies in the computational languages and frameworks, statistics and mathematics, run-time schemes, operating schemes, compilers and algorithms, application models, software tools, middleware, advanced hardware and virtual machines.

- Secondly, it is based on the application Research and Development (R\&D) in the usage of high-end computational resources in collaboration with scientific domains, incorporating novel computing, statistical and mathematical modeling, analytic tools, visualization tools, aggressive domain-based applications and deployment of scalable information management schemes.

- Thirdly, it is based on the construction, deployment and testing of both the innovative and sustainable resources into the collaborative environment, which integrates the coordination and integration of resources. 
- Fourthly, the development of critical workforce and educational programs, from deep professionals in statistical, mathematics and computational simulations, CDE\&S and modeling to structure enabling career opportunities and technical workforce in industry, government, academic and science.

- The evaluation and development of grand and transformational challenge society programs, which support more contemporary and complex issue-solving through the integration of integrated and compressive strategies for science, networking, software, facilities, high-end computing, transnational partnerships and governmental agencies.

Section I has introduced the concept of Advanced Computing Infrastructure (ACI) Strategic Plan, its vision and strategies. The remaining sections of this articles focus on further exploiting the concept with further emphasis on the Cyber Infrastructure (CI) of the 21st Century and its framework integrate other key complementariness. In that regarding, the remaining part of the paper has been organized as follows: Section II presents a background analysis of the research. Section III analyses the relevant literature works. Section IV presents a critical analysis of the CI and the strategic directions of ACI. Lastly, Section V concludes the paper and provides future research directions.

\section{BACKGROUND}

Innovative data initiatives are rapidly changing the fabric of the community and information in the novel currency for commerce, government, education and science. High-performing computing is vital for the establishment of fundamental modeling and simulation as the $3^{\text {rd }}$ science pillar (experiments and theories considered to be the $1^{\text {st }}$ two), and the developing significance of information is forming the $4^{\text {th }}$ pillar. The progressive development in the various cores in every chip and accelerator-centered hybrid schemes necessitate diversified R\&D efforts in the novel computing systems, computational frameworks, parallel programming language and software enhancement for distributed and parallel systems. This also facilitates more attentions for the resiliency (fault-tolerance), novel operating schemes, and the runtime schemes.

Energy consumption is presently a fundamental limitation for the various dimensions of computers. Similarly, the bandwidth of memory limitation and enhanced information movement necessitates fundamental efforts in research efforts in statistical sciences, computer science, engineering sciences, material sciences and mathematical science. The interdisciplinary analysis and application of data necessitate increased dimensions of $R \& D$ in advanced simulation approaches, coupling more complex frameworks, novel algorithms, strategies to software and data resilience and integrity, novel statistical tools and data analytics, including data sustainability and data management. The development of data-intensive science integrated with interdisciplinary collaborations integrate more efforts in the novel and existing domain-based tools and applications integrating mathematics, statistics and software engineering, widening the application of computing across the wide-range NSF, and structuring the complete CDE\&S workforce.

According to the Presidential Council of Advisors concerning Technology and Science, formulating the digitalized future points out that the floating operations in every second measures are not considered as definitive measurements for high-performing computing success and that it is presently significant to conduct more basic researches in hardware, software/hardware schemes in algorithms and in the various applications and system software [2]. High-performing computation has to integrate the capacity to fundamental manage and manipulate diversified amounts of data. It also has to simultaneously focus on the initiates in algorithms and software applications, statistical methodologies, data analytics, critical operating system analysis, innovative domain-centric application and the file systems.

The novel ACI strategies, in this paper, focus on the problems presented by the report made by the Presidential Council of Advisors concerning Technology and Science, commoditization of both the software and hardware is formulating an era of fundamental disruptions. The $1^{\text {st }}$ disruption is the transforming role and nature of the private department in the development of the upcoming generation of technology and computing. Advanced computing and big data will not be governed by higher-end scientific protocols, but based on wide-range $\$ 1000$ devices (tablets, cell phones, and computer games). The micro-scale margin will stimulate manufacturers towards the volume, and novel technologies will be left out in case they do not integrate more demonstrable market shares required to recoup any production costs and development costs.

The $2^{\text {nd }}$ disruption originates from the ideology that ubiquitous presence of wide-range technologies will incredibly transform the development of multiple workflows and processes, integrating the type of software an algorithms, which have to be implemented in education and research. The $3^{\text {rd }}$ disruption is the introduction of the facilities incorporated in the higher education department, since there is an incredibly less linkage between the physical places in institutions and researchers [3]. This will amount to the novel frameworks for data-centric sciences, which will be organized in a dynamic manner around the research domains and questions, and will also present novel issues to geographically-based R\&D efforts, integrating contemporary educational institutions.

Cyber Infrastructure (CI) is a complex and emerging concept. To provide a detailed background, this paper draws definitions from NSF reports, social scientists and technologists who have written documents on CI. We start with the documents, which stimulated the NSF to establish the CI office. The report defines infrastructure based on the distributed computing, communication technologies and information. The base initiatives underlying CI include the integrated electro-optical elements of communications, storage and computation, which progresses on to advance in the raw capabilities at a dynamic rate. The literature review (in Section III) below focuses on the previous works on CI with details about the CI layers, technologies, activities and CI application. 


\section{LITERATURE REVIEW}

H. Kiertzner in [4] evaluated the CI layers and the elements above them. It was noted that above the CI layer, there are instruments, information, data, knowledge, software programs, social programs and services applicable to particular projects, societies of practice and disciplines. Between the two layers is CI layer of personnel, institutions, communications, software, algorithms, and enabling hardware. The layer has to provide an efficient and effective platform that empowers particular societies of analysts to innovate and definitely revolutionize the activities they do, the manner in which they do them, and the people involved.

Y. Xia, T. Zhang and S. Wang in [5] presented a definition of Cyber Infrastructure (CI) and the definition concludes a focus on the empowerment of the research society to dynamically generate a revolution on engineering and science. The concept is defined as a platform consisting of data repositories, people, visualization ecosystems, advanced instruments, data storage schemes and computing systems all connected by high-performance network and software to enhance the productivity of research and enhance breakthroughs that were considered impossible. Even though the researchers focus on the technical element of CI, they suggested that 'people' play a fundamental role in the significantly technical structure. Adding to the specialized initiatives, it is suggested that CI also integrates organizational and personal webpages with data and information, net meetings, email communication and common search engines e.g. Google and digital libraries.

R. Harris in [6] argue that the integration of technologies suggest that a fundamental concept of CI and the science driven by $\mathrm{CI}$ is to be governed by daily communication and business activities engaged by the people incorporated in it. Whereas CI is considered more technical in nature, the issues from its non-technical aspects have long been identified. The researchers argued that "softer" foundations of CI might be a challenging issue to fundamentally facilitate CI-driven collaborations. It was argued out that the success in realizing full potential in e-science and the relevant transnational collaborative activities have been supported by CI, if they are to be attained, and will likely be resultant of the associative nexus of technical, legal and social transformations. This means that it is potentially "softer" parts (i.e. non-engineering) and every bit is as complicated as the computer software and hardware, and actually, might be harder to implement or devise.

K. Michael in [7] recognized the business and social challenges of CI. The challenges attracted the attention of researchers to brainstorm about the human aspects of CI, including its infancy, so the efforts and investments, which are dedicated to its development, are not proved futile. This is towards producing sophisticated analytic lens to comprehend the organizational and social complexity of CI.

M. Likosky in [8] proposed the Human Infrastructure (HI) concept and defined it as the organization of actors and businesses, which have to be brought into the alignments for wok to be completed. The researchers further elaborated that the $\mathrm{HI}$ in larger CI projects is a significant series of overlapping contemporary entities, consortia, loosely arranged in networks and groups. In the seminal piece on illustrating the aspect of HI of CI, they concentrated on the description of the social status and the activities, which integrate the advent of infrastructure in geoscience resonances. The principal issues are minimally technical compared to organizational, social and cultural. Before data can be made interoperable, we should make people more interoperable.

H. Tiirmaa-Klaar in [9] proposes and synthesizes the definition of CI to be data intensive, large-scale, computationally powerful, interoperable, hierarchical and integrated with the second-order development over a considerable time-frame. It integrates a general and specialized hardware, high-performing computing applications, data and communication initiatives, both human and non-human agents, connective and interacting via the interdimensional network. The platform stimulates technologically-produced virtual ecosystems and socially-produced virtual entities, which orient technology, data and people towards standard goals.

A. Djenna, S. Harous and D. Saidouni in [10] evaluate the CI amounts to enhanced productivity, paradigmatic revolution, and breakthrough innovation. Simplistically, CI represents an empowering network of more advanced technologies, collaborative groups/people and metadata. This synthetic illustration indicates the manner in which CI operates as a socio-technical scheme of technology and people, which is efficient only when it potentially interacts. Provided the article's constant focus on CI (other than collaborator, e-science, etc.), a possible risk is to appear as technologically deterministic in our perspective. The researchers maintained that it is possible to put more emphasis on the materiality of organizations without considering being technologically deterministic. We regard both the material and human dimensions of CI similarly fundamental.

This paper focuses on the human dimensions in the interplay between material technology and social arrangement in the aspect of CI. In that regard, we switch to the discussions regarding the features we argue to link with literature in organizational and communication studies. Firstly, researching CI diffusion and adoption aids in the description of the future large-scale data infrastructure. The movement of CI from the grassroots society efforts towards an organized and federally funded programs e.g. the cases of XSEDE and TeraGrid in America, recommends that the movement of CI might be transforming from the bottom-up efforts into more top town programs. Therefore, the diffusion and adoption analysis can be noted from the emerging dimensions of implementation and dissemination science about constructing and sustaining large-scale R\&D efforts and programs. Section IV below provides a critical analysis of what has been introduced in this paragraph. Furthermore, the section below presents a critical analysis of the vision and strategic directions of the CI framework of the 21 st Century 


\section{CRITICAL ANALYSIS}

\section{Studying Cyber Infrastructure (CI) Adoption and Diffusion}

Dissemination is an active strategy with the obligation of spreading technologies to target audience through determined channels using planned approaches. Implementation evaluates "what typically takes place after a specific adoption has been made, mostly in the business setting. Implementation is one phase (after adoption and awareness and before sustained application) in the over-time diffusion process. Recently, CI is shifting from an innovative and organic organization (bottom-up, "nomadic science") to the centralized and controlled organization (top down, "royal science"). The observation signals a requirement to shift beyond what researchers illustrate as uncontrolled, unplanned, untargeted and passive innovation diffusion to what we might purpose to argue for: a controlled, planned, targeted and active program of CI implementation and dissemination. Provided Cl's bespoke/participatory and revolutionary/disruptive features, one potential fruitful study focus is on community, organizational and individual for implementation and adoption towards constructing a future large-scale data infrastructure.

Secondly, evaluating CI adoption and diffusion provides us with a sense of the upcoming large-scale communications network. CI advent out of the inter-business consortia, collaborations, communities, including the complex/meta-complex and network/community features suggest that $\mathrm{CI}$ can present a case for evaluating the recursive connections between micro interactions and macro structure layers in a massive society of practices and more complex systems. In simple terms, the sociological structural theory can possibly shed enough light on the adoption strategies and diffusion procedures in the communications networks of CI. Specifically, one possibly applicable strategy is to conceptualize three dimensions of communication networks and recursive connections: people and businesses, businesses and communities/consortia, and communities/consortia and people. This strategy can consider framing the whole context of CI as a novel moral society with more complex networks.

In addition, this strategy can incorporate the ideology of sequential and concurrent application of technologies to accomplish the required works. This strategy applies both the structural ideology and technology sequences/concurrencies more completely capturing the complexities of the networks and relationships, and the manner in which adoption and diffusion analyses can take more complex contexts of networks, consortia and organizations into comprehending people, inter-organizational and organizational adoption choices in more complex environment of media and technologies.

Thirdly, a study of CI adoption and diffusion impacts enough understanding of the adoption and diffusion of its interlinked organizational and social practices in form of upcoming massive scale virtual entities. Revolution does not take place when the community adopts novel technologies. Otherwise, it takes place when the community adopts novel behaviours. For CI to revolutionize engineering and science, a key NSF item represents its funding objective is to develop efficient virtual businesses facilitated by CI. This is the case in NSF's creation of the Virtual Organizations as Social-technological System (VOSS) program under the CI office in 2008.

R. WANI and H. IKEDA in [11] utilize the terminology "flow" as a critical data analytical framework to comprehend the critical forces, which define the modern society. The study argues that several flows integrating fiscal capital, labor immigrations, financial capital foreign, technological expertise, information, production and culture among others, provide a rise to networked community we dwell in. Similarly, M. Bogdanowicz in [12] utilizes similar terminology to evaluate the communicative organizational constitution. It is argued, in their research, that the membership negotiations, activity coordination, institutional positioning and self-structuring are the four fundamental communicative flows, which provide a rise to businesses.

As for $\mathrm{CI}$ and its interlinked virtual businesses, the membership negotiations denote to who are viewed as the members of CI projects and the ones who have accessibility to the CI resources from throughout the country through the web. Self-structuring denotes to the CI guidelines and program proposals, which aid in the numerous participants, large societies and distributed entities to custom-structure towards the stated objectives. The coordination of activities denotes to what the distributed participants and virtual teams to on normal basis to effectively coordinate their CIbased activities, sometimes shifting from what one can do if acting from the general based on custom-structure documents and guidelines. Institutional positioning is the manner in which organized teams, virtual entities, larger societies and funding agencies collaboratively communicate CI to external audience, integrating the congress, the public and the research community. Another possible direction, in addition to the 4 flows, is to study the manner in which McPhee's 4 flows pairs and overlaps hence providing a rise to entities.

Vision and Strategic Directions of Advanced Computing Infrastructure (ACI)

Whereas supercomputers are still considered the major generators of data, the exponential increment in data from the advancing distributed collection of diversified scientific tools and the sensors networks necessitate a novel and qualitatively varied strategy to information storage, management, access, distribution, Cyber Security (CS) and stewardship. Not only is the information massive, diversified and distributed, but the requirement for data evaluation necessitates possibly varied statistical, mathematical and computational strategies and the collaborative nature of study have enhanced the requirement for more distributed accessibility. This novel ACI vision by NSF supports both data-intensive and computational research originating from the scientific tools, simulations, sensors and cloud computing. It is fundamental that the newly formulated ACI environment can accommodate contemporary local centers including those available at Universities, and integrate local clusters, supercomputers, visualization schemes, and storage schemes, which are capable of supporting more researchers compared to the past. 
Sustainable research and advanced technologies in high-performing computing has formulated ubiquitous requirement for more advanced digital services over multiple landscapes, from campuses and schools to research communities and industries. In that regard, the vision of NSF for advanced computing also should diversify the major focus on wide basis of CDE\&S over multiple domains. Accomplishing ACI vision will diversify engineering and science research and studying to serve the local need for decades to come. NSF ACI approaches a major segment of the massive NSF CI of the 21st Century and is not stand-alone and separate efforts (see Fig 1). Despite the fact that this paper focuses on the ACI-particular strategies, it is fundamental to consider that the complete CI of the $21 \mathrm{st}$ Century planning integrates an integrative strategy to effectively support the issues and problems addressed by education communities, engineering and science.

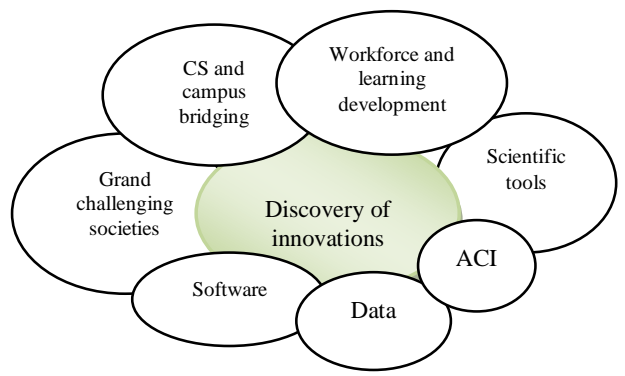

Fig 1. CI framework of the 21st Century

Firstly, Basic research to complely exploit the concurrency and parallelism via innovations in computational languages and frameworks, statistics, mathematics, run-time and operating systems, compilers, software instruments, middleware, application models, advanced hardware and virtual machines. This strategy integrates:

- Computational frameworks to faciliate novel and transformative strategies of "brainstorming parallel" integrating novel abstractions, which account for concurrency and parallelism, and support reasoning cocnerning the parallel and correctness performances, integrating communications, security, resiliency and energy costs.

- Programming language to enahnce efficient expression of concurrency and parallelism at each scale, integrating novel strategies to develop scale, handling texts and shared memory, and enhancing the productivity of programming on distributed and parallel systems.

- Disruptive rethinking of cononical computing stack - architecture, operating systems, virtual machiines, run-time system, compilers, programming languages and application - based on resource-management and parallelism problems and for supporting optimization across the various dimensions of the stack from the architecture level to the software down.

- Novel paradigms of the algorithms, which promote reasoning about the parallel performance and amount to provable performance assurance, whereas permiting algorithms to be mapped effectively onto the diversified distributed and parallel ecosystem and and optimizing the usage of resources integrating Input/Output, Communication, Compute Cycles, Energy and Memory Hierarchy.

- Computer software system to enhance resilient computatio at a larger scale, integrating novel operating schemes for multicore schemes and the cloud systems; data stores and file systems for data-intensive computing, run-time schemes to effectively manage parallelism, communication, synchronizaiton, energy usage, scheduling and compilers meant too manage security, energy consumption, predictability and debugging;

- Computer architecture, which concentrates on effective communicaiton, integrating multiconnection networks, parallel memory schemes, fine-grain synchronization and input/out;

- Research analysis into the significantly scalable and parallel application-based and heterogeneous scheme architecture;

- Software architectures and algorithms able to handle both small-scale and large-scale data analytics and data systems;

- Critical research in methodologies, statistical theories and mathematical algorithms to focus oon the potential issues with distributed and big data.

Secondly, R\&D in the application of high-end computing resources in collaboration with the scientific domains, integrating novel mathematical, computational, and visualization, simulation, statistical model and aggressive domain-based application developmennt, data analytical tools, deployment of more scalable schemes for data management. This strategy integrates:

- The systematic exploration of the upcoming generation of scientific approaches, applications, and algorithms in the various disciplines, their computational requirements, and their mapping mapping concerning the potentual future approaches and architectures to the aspect of computing; 
- Novel algorithms meant to effective exploit significantly distributed and parallel platforms, and for dataintensive computational obligations, including the approaches to effectively decompose the present serial algorithms into rapid integration of distributed, parallel and serial computation [13];

- Research into the significantly scalable and parallel application-basic and heterogeneous systems;

- Focussed investments into the algorithmic development, software and tools, which will effectively support the various disciplines, mostly the ones that have not used concurrency and parallelism capabilities in the past, integrating scientific approaches for statistical evaluation, simulation, visualization and data mining, including sophisticted CI for discipline-centered scientists (eg education researchers, economists, social scientists, geologists, and biologists) [14];

- Integrated data (end-to-end) pipeline paradigm of management to harness concurrency and parallelism, concentrated on the complete data path from its generation process to the transmission process, to storage, application and maintainability, all the way to definitely destruction or archiving;

- Developing sustainable data services meant to provide data mining, mathematical algorithms, computational tools and statistical evaluations to a wider collection of educators, scientists and researchers, and hence boosting the research across significant segment e.g. education, engineering, computational sciences, mathematics, and data statistics.

Thirdly, constructing, testing and deploying both innovative and sustainable resources into a more collaborative environment, which integrates coordination/integration with regional and campus systems, cloud services, networks, and data centers in collaboration with scientific domains. This strategy integrates:

- Sustainable and balanced strategy to NCF ACI services, integrating support for not just high-performing computing hardware, but also for a wider culture of scientific computing aid and integrating methodologies, which are beyond contemporary high-performing computing services integrating the involvement with national computational resources and campus resources, and an exploration of the advancing number and capacities of the cloud services and systems. This strategy will also incorporate a close working connection with universities. A development of high-performing computing services to be supporting of the significant data centers being established by the scientific domain, and enhance the storage of legacy data and permit societies to integrate and access such sets of data in means that are presently not possible;

- Developing the capacities, which concentrate on ACI for the wider research and science community, which integrate the facilities, which all the researchers can utilize and support their staff who might be available and trained for consultation, including the strategic investments in the domain-based ACI centers;

- Revising the present allocation processes to effectively accommodate a significantly array of disciplines, effective incorporation with the school infrastructure, and the allotment of data resources and storage schemes;

- The development of more sustainable CI incorporating high-speed, transmission (end-to-end) with data management, storage and curation to effectively support societies in doing data-specific sciences (such as earth system model, astrophysics, astronomy, health-informatics, social schemes, economics, molecular modeling, biodiversity informatics, phenomics, phylogenomics, and genomics;

- Alignment of plans for data infrastructure with CI plans.

Fourthly, the development of quality workforce programs and education programs, from the construction of deep expertise in statistical, mathematical and computational simulation, modeling, and CDE\&S meant to effectively develop more technical workforce and the enabling career opportunities in industries, governments, academia and science. This strategy incorporates:

- Workforce and educational development is required to effectively support the upcoming generation of applied and computational sciences as ACI, computational and data-oriented sciences that go mainstream. These fundamental efforts might integrate research experiences for the undergraduates, advanced technological education, graduate research fellowship, faculty early careers, postdoctoral and modeling; evaluation of the required expert skillset and capacities in CDE\&S, integrating computational science professional obligations and the investments in developing the learning progression to inform programs and curriculum to develop that skillset. Developing career activities, program development and curriculum development focused on serving these needs should involve the evaluation of novel and emerging expert duties and the required preparation and training. These efforts have to be centered on developing enough evidence concerning efficient learning of novel, and complex domains;

- Development of novel and diversified course curricula and career educational resources for distributed and parallel computing languages, data analytics and data-intensive sciences, again at the dimensions that range from the post-doctoral professions to experts in education, engineering and sciences to the preparation of experts;

- Expansion and adaptation of the present programs, such as such as scholarship of services, mathematics, Science, Technology, Engineering and Mathematics (STEM), incorporative graduate research and 
education focused on the development of career opportunities and workforce programs for undergraduate and college education, multi-disciplinary and applied experience at the post-doctoral and graduate levels;

- Novel research into the manner in which people study the concepts of concurrency parallelism; R\&D of efficient approaches to train distributed and parallelism computing and data-oriented sciences and simulation;

- Investing in graduate and undergraduate curriculum development and education, which will effectively prepare the upcoming generation of disciplinary professionals to effectively engage in science with fundamental CDE\&S and computational levels, integrative the major focus on the obligation of apprenticeship and practical experiences;

- Focus on widening the participation levels, novel approaches for recruitment to the undergraduate programs in this segment, and the formulation of senior leadership talents areas.

Fifthly is the development and assessment of grand and transformational challenge society programs, which support more traditional complex issue-solving process by involving the integrated and compressive methodology to science, using high-end computing, networking, data, software, facilities, multidisciplinary professionals across societies, other international partnerships and governmental agencies. This strategy integrates:

- Novel focus on the grand and transformational challenge societies will build on the enabling investments in terms of infrastructure (such as Stampede, Facilities, Instruments and Blue waters, Data service), institutes of software programs, core technologies (novel algorithms and approaches), CDE\&S (supporting personal investigators or the smaller teams in critical strategies to CDE\&S), workforce and learning (to effectively develop a novel generation of computing researchers), sharing the interdisciplinary data over wide-range institutions and government agencies to enable the communities and teams to effectively address the upcoming generation of major professional challenges;

- On the integrated ecosystem, the programs will integrate the formation and development of detailed, interdisciplinary programs meant to support communities and teams to attack more complex and transformational engineering and science problems, necessitating integrative strategies to data, computation, hypothesis testing, which cannot be addressed effectively by a smaller group, and necessitating the groups, which integrate domain engineering and science, alongside enabling sciences;

- More investments and focus on the long-lasting sustainability of research groups to effectively address what are normally decadal efforts for grand problems;

- Investments meant to facilitate the formation of interdisciplinary professionalism in collaboration with schools as the core competency for studies and to formulate CDE\&S as a fundamental career path.

Implementation of the ACI strategies dovetails and complements with other CI of the 21 st Century elements, integrate CS, workforce development, learning, software, data, and custom directorate, education efforts and office research. CDE\&S and the grand issue communities' activities interlink with the ACI and the various elements of the $\mathrm{CI}$ of the 21st Century strategy. The activities are enabled and stimulated by the coherent strategy to formulate these elements to accomplish the science and research needs of the community. A detailed evaluation of the strategic directions has been provided in the paragraphs above.

\section{CONCLUSION AND FUTURE DIRECTIONS}

The capabilities of advanced computing are applied in handling the significantly developing array of problematic engineering and science challenges, most of which are data-intensive and computational. The growing demand of advanced computing for the various capabilities and types of systems, from larger numbers of commodity nodes to the tasks necessitating thousands of cores; for the schemes with prompt connects; for the schemes with smart data management and handling; and for increasingly diversified collection of application that incorporate data analytics, including simulation and modeling. Since the introduction of supercomputers, NSF has issued its experts with sophisticated computing schemes. The advancement of the novel frameworks in computing, integrating cloud computing and locally-present by privately reserved repositories creates more possibilities for NSF. To effectively comprehend the diverse and expanding needs of the engineering and science community and the significance of a novel range of ACI, it is fundamental to undertake research to evaluate the tradeoffs and priorities for advance computing. This future research direction is meant to provide a sophisticated framework for decision-making about the advanced computing programs and strategies for the upcoming generations.

\section{References}

[1]. N. Nor, A. Miskon, Z. Yunos, M. Ahmad and A. Zaidi, "Cyber Force Establishment: Defence Strategy for Protecting Malaysia's Critical National Information Infrastructure Against Cyber Threats", Global Security and Intelligence Studies, vol. 4, no. 2, 2019. Available: 10.18278/gsis.4.2.4.

[2]. R. McDonald, "Science Ph.D. and Science Postdoctoral Advisors for the 21st Century: A Path To Successful Technology Transfer", Technology Transfer and Entrepreneurship, vol. 1, no. 1, pp. 40-50, 2014. Available: 10.2174/2213809901666140110234348.

[3]. S. Gottlieb, "The Future of NSF Advanced Computing Infrastructure Revisited", Computing in Science \& Engineering, vol. 18, no. 5, pp. 4-7, 2016. Available: 10.1109/mcse.2016.85.

[4]. H. Kiertzner, "Cyber security for critical national infrastructure", Engineering \& Technology Reference, 2016. Available: 10.1049/etr.2016.0004. 
[5]. Y. Xia, T. Zhang and S. Wang, "A Generic Methodological Framework for Cyber-ITS: Using Cyber-infrastructure in ITS Data Analysis Cases", Fundamenta Informaticae, vol. 133, no. 1, pp. 35-53, 2014. Available: 10.3233/fi-2014-1061.

[6]. R. Harris, "New technologies and data integration", Space Policy, vol. 16, no. 1, pp. 77-78, 2000. Available: 10.1016/s02659646(99)00050-8.

[7]. K. Michael, "Handbook on Securing Cyber-Physical Critical Infrastructure: Foundations and Challenges", Computers \& Security, vol. 31, no. 8, p. 1013, 2012. Available: 10.1016/j.cose.2012.07.007.

[8]. A. Haldorai and A. Ramu, "Security and channel noise management in cognitive radio networks," Computers \& Electrical Engineering, vol. 87, p. 106784, Oct. 2020. doi:10.1016/j.compeleceng.2020.106784

[9]. A. Haldorai and A. Ramu, "Canonical Correlation Analysis Based Hyper Basis Feedforward Neural Network Classification for Urban Sustainability," Neural Processing Letters, Aug. 2020. doi:10.1007/s11063-020-10327-3

[10]. M. Likosky, "Human Rights Risk, Infrastructure Projects and Developing Countries", Global Jurist Advances, vol. 2, no. 1, 2002. Available: 10.2202/1535-1661.1051.

[11]. H. Tiirmaa-Klaar, "Building national cyber resilience and protecting critical information infrastructure", Journal of Cyber Policy, vol. 1, no. 1, pp. 94-106, 2016. Available: 10.1080/23738871.2016.1165716.

[12]. A. Djenna, S. Harous and D. Saidouni, "Internet of Things Meet Internet of Threats: New Concern Cyber Security Issues of Critical Cyber Infrastructure", Applied Sciences, vol. 11, no. 10, p. 4580, 2021. Available: 10.3390/app11104580.

[13]. R. WANI and H. IKEDA, "Planispiral Cephalopod Shells as a Sensitive Indicator of Modern and Ancient Bottom Currents: New Data from Flow Experiments with Modern Nautilus pompilius", PALAIOS, vol. 21, no. 3, pp. 289-297, 2006. Available: 10.2110/palo.2004.p04-92.

[14]. M. Bogdanowicz, "The Communication Audit: Information Flow and the Organization", Discourse and Writing/Rédactologie, vol. 10, no. 1, p. 10-Jan, 1992. Available: 10.31468/cjsdwr.325. 